\title{
An atomistic perspective on the intrinsic white-light photoluminescence of rare-earth free $\mathrm{MgMoO}_{4}$ nanoparticles
}

Anderson A.G. Santiago ${ }^{* a}$, Marisa C. Oliveira ${ }^{\mathrm{a}}$, Renan A.P. Ribeiro ${ }^{\mathrm{b}, \mathrm{c}}$, Ricardo L. Tranquilin ${ }^{\mathrm{b}}$, Elson Longo ${ }^{\mathrm{b}}$, Sergio R. de Lázaro $^{\mathrm{d}}$, Fabiana V. Motta ${ }^{\mathrm{a}}$, Mauricio R.D. Bomio*a

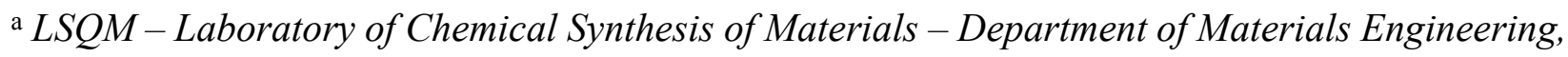
Federal University of Rio Grande do Norte-UFRN, P.O. Box 1524, Natal, RN, Brazil

b CDMF-UFSCar, Universidade Federal de São Carlos, P.O. Box 676, 13565-905 São Carlos, SP, Brazil

c Department of Chemistry, Minas Gerais State University, Av. Paraná, 3001, 35501-170, Divinópolis, MG, Brazil

${ }^{d}$ Department of Chemistry, State University of Ponta Grossa, Av. General Carlos Cavalcanti, 4748, 84030-900, Ponta Grossa, PR, Brazil.

*Corresponding authors. E-mail address: andersonsantiago@ufrn.edu.br (Santiago, A.A.G.), mauricio.bomio@ct.ufrn.br(Bomio,M.R.D). 


\section{Supporting Information}
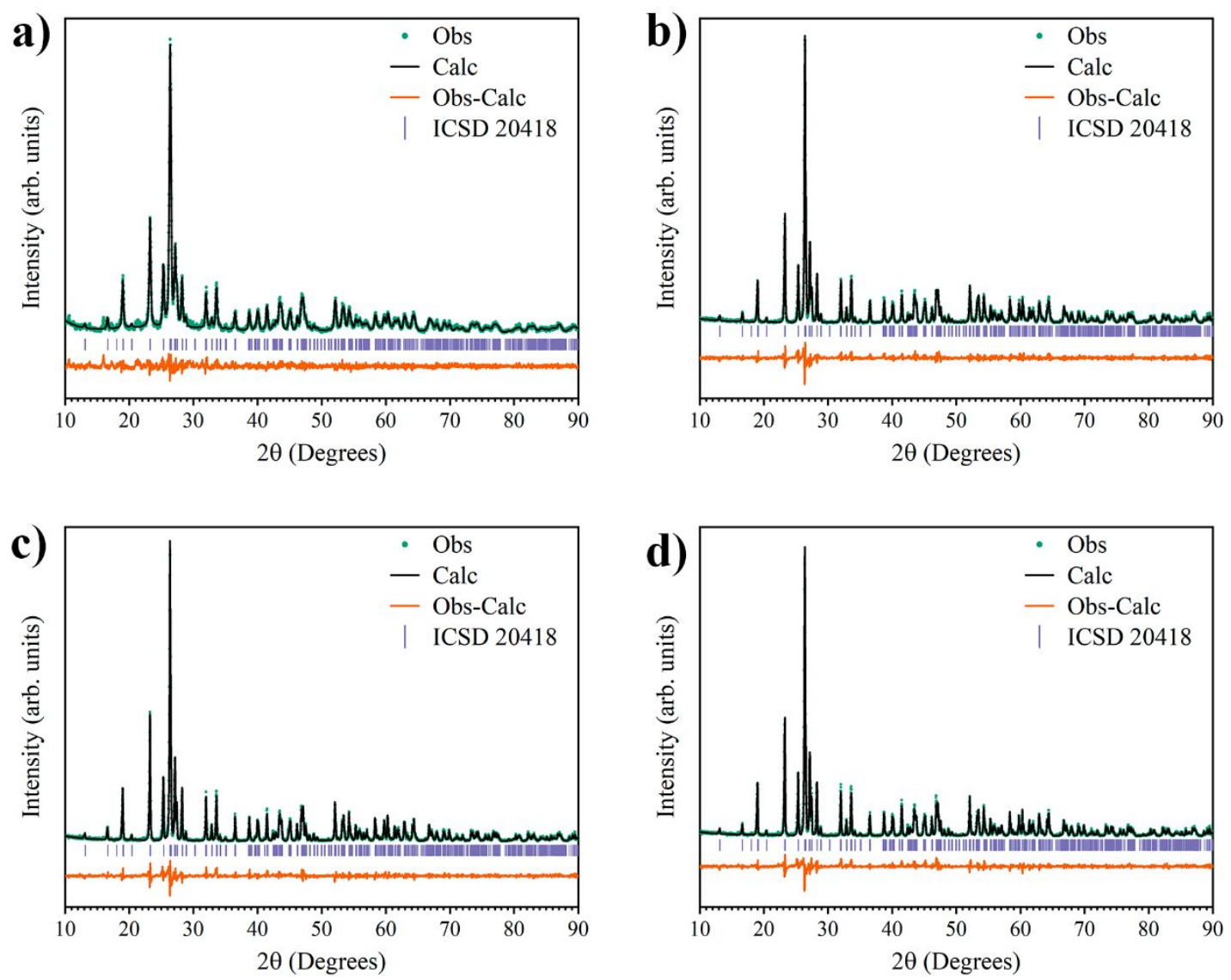

Figure S1. Rietveld refinement patterns for $\mathrm{MgMoO}_{4}$ samples, namely: (a) MgSpray, (b) Mg800, (c) Mg900, and (d) Mg1000. 


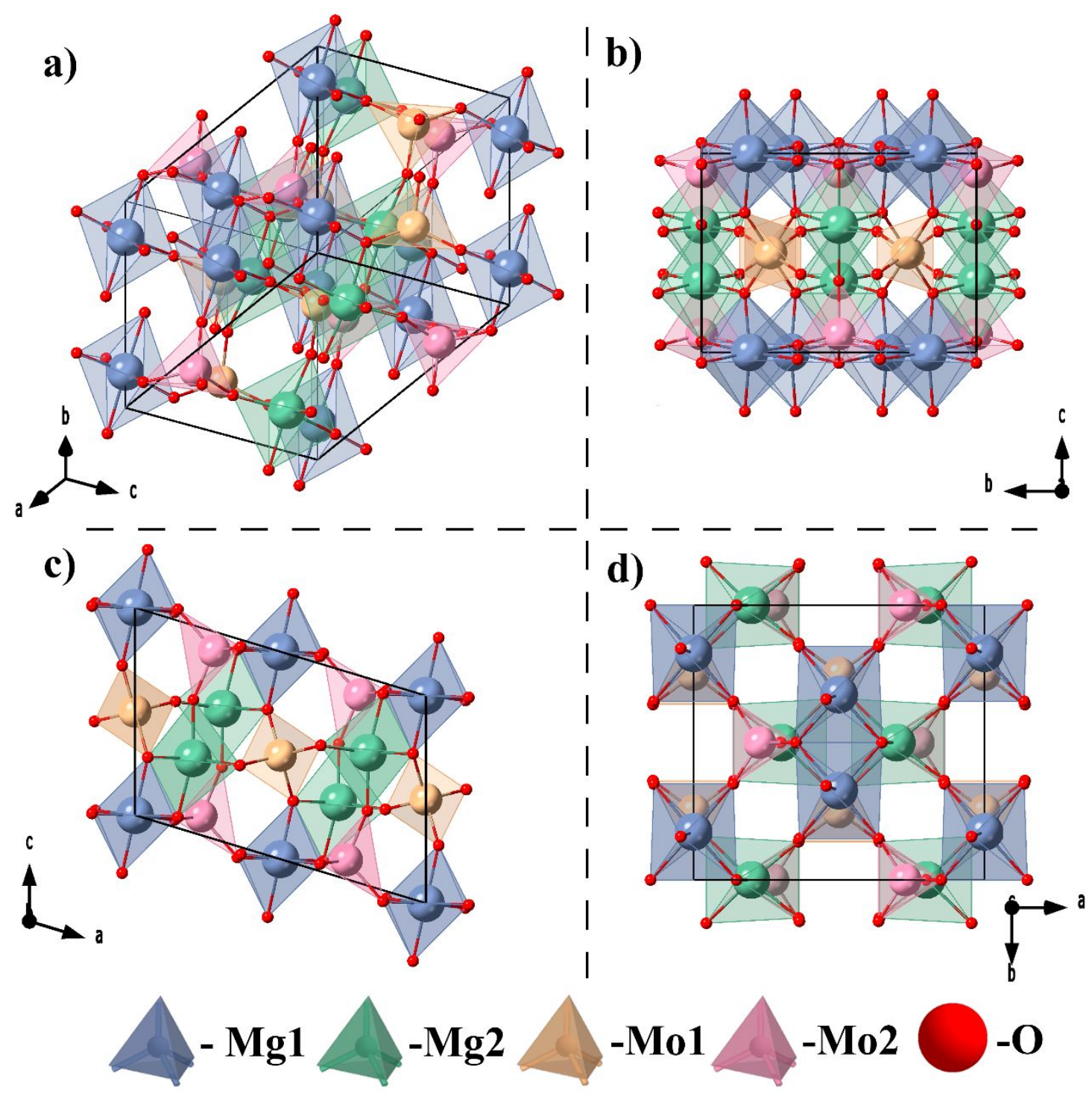

Figure S2. Schematic crystal structure of $\beta-\mathrm{MgMoO}_{4}$ from the (a) standard orientation of crystal shape, (b) a-axis direction, (c) b-axis direction, and (d) c-axis direction. 
Table S1. Fractional atomic coordinates of $\mathrm{MgMoO}_{4}$ samples.

\begin{tabular}{ccccccc}
\hline Sample & Atom & $\mathbf{x}$ & $\mathbf{y}$ & $\mathbf{z}$ & Occ. & $\mathbf{U}$ \\
\hline \multirow{5}{*}{ MgSpray } & Mo1 & 0.000 & 0.252 & 0.500 & 1.000 & 0.026 \\
& Mo2 & -0.274 & 0.000 & 0.091 & 1.000 & 0.025 \\
& Mg1 & 0.000 & 0.824 & 0.000 & 1.000 & 0.035 \\
& Mg2 & 0.198 & 0.000 & 0.363 & 1.000 & 0.028 \\
& O1 & 0.543 & 0.658 & 0.300 & 1.000 & 0.025 \\
& O2 & -0.859 & 0.651 & 0.039 & 1.000 & 0.025 \\
& O3 & -0.862 & 0.357 & 0.604 & 1.000 & 0.025 \\
& O4 & 0.648 & 0.500 & 0.059 & 1.000 & 0.025 \\
& O5 & 0.203 & 0.000 & 0.642 & 1.000 & 0.025 \\
\hline & Mo1 & 0.000 & 0.253 & 0.500 & 1.000 & 0.018 \\
& Mo2 & -0.272 & 0.000 & 0.095 & 1.000 & 0.016 \\
& Mg1 & 0.000 & 0.822 & 0.000 & 1.000 & 0.017 \\
& Mg2 & 0.197 & 0.000 & 0.354 & 1.000 & 0.012 \\
Mg800 & O1 & 0.546 & 0.651 & 0.295 & 1.000 & 0.025 \\
& O2 & -0.866 & 0.660 & 0.037 & 1.000 & 0.025 \\
& O3 & -0.855 & 0.360 & 0.613 & 1.000 & 0.025 \\
& O4 & 0.646 & 0.500 & 0.051 & 1.000 & 0.025 \\
& O5 & 0.210 & 0.000 & 0.641 & 1.000 & 0.025 \\
\hline & Mo1 & 0.000 & 0.252 & 0.500 & 1.000 & 0.024 \\
& Mo2 & -0.271 & 0.000 & 0.096 & 1.000 & 0.023 \\
& Mg1 & 0.000 & 0.823 & 0.000 & 1.000 & 0.029 \\
& Mg2 & 0.199 & 0.000 & 0.356 & 1.000 & 0.022 \\
Mg900 & O1 & 0.543 & 0.652 & 0.297 & 1.000 & 0.025 \\
& O2 & -0.864 & 0.656 & 0.037 & 1.000 & 0.025 \\
& O3 & -0.859 & 0.353 & 0.614 & 1.000 & 0.025 \\
& O4 & 0.649 & 0.500 & 0.051 & 1.000 & 0.025 \\
& O5 & 0.209 & 0.000 & 0.647 & 1.000 & 0.025 \\
\hline Mo1 & 0.000 & 0.252 & 0.500 & 1.000 & 0.020 \\
& Mo2 & -0.271 & 0.000 & 0.095 & 1.000 & 0.019 \\
& Mg1 & 0.000 & 0.822 & 0.000 & 1.000 & 0.023 \\
& Mg2 & 0.198 & 0.000 & 0.354 & 1.000 & 0.017 \\
& O1 & 0.546 & 0.649 & 0.297 & 1.000 & 0.025 \\
& O2 & -0.866 & 0.657 & 0.036 & 1.000 & 0.025 \\
& O3 & -0.860 & 0.360 & 0.611 & 1.000 & 0.025 \\
& O4 & 0.645 & 0.500 & 0.048 & 1.000 & 0.025 \\
& O5 & 0.200 & 0.000 & 0.645 & 1.000 & 0.025 \\
\hline & & & & & & \\
& &
\end{tabular}


Table S2. Theoretical and experimental values of the Raman frequencies calculated and experimental of $\mathrm{MgMoO}_{4}$ crystal. The symbols $\mathrm{B}, \mathrm{S}$ and $\mathrm{R}$ correspond to Bending, Stretching and Rotational type of motions.

\begin{tabular}{|c|c|c|c|}
\hline \multirow{2}{*}{ Raman mode } & \multirow{2}{*}{ Type of motion } & \multicolumn{2}{|c|}{ Wavenumber $\left(\mathrm{cm}^{-1}\right)$} \\
\hline & & Theoretical & Experimental \\
\hline$B_{g}$ & $\mathrm{R}$ & 34.63 & - \\
\hline$B_{g}$ & $\mathrm{R}$ & 66.82 & - \\
\hline$A_{g}$ & $\mathrm{R}$ & 91.05 & - \\
\hline$B_{g}$ & B & 95.27 & - \\
\hline$B_{g}$ & B & 109.73 & - \\
\hline$A_{g}$ & B & 125.77 & 121 \\
\hline$B_{g}$ & $\mathrm{~B}$ & 126.98 & - \\
\hline$A_{g}$ & B & 157.22 & 155 \\
\hline$B_{g}$ & B & 168.75 & - \\
\hline$A_{g}$ & B & 180.92 & 178 \\
\hline$B_{g}$ & B & 207.74 & 203 \\
\hline$A_{g}$ & B & 217.14 & - \\
\hline$A_{g}$ & B & 275.86 & 275 \\
\hline$B_{g}$ & B & 283.29 & - \\
\hline$B_{g}$ & B & 288.21 & - \\
\hline$B_{g}$ & B & 305.06 & 303 \\
\hline$A_{g}$ & B & 305.86 & 300 \\
\hline$A_{g}$ & $\mathrm{~S}$ & 331.67 & 321 \\
\hline$B_{g}$ & $\mathrm{~S}$ & 332.97 & 334 \\
\hline$A_{g}$ & $\mathrm{~S}$ & 337.83 & - \\
\hline$A_{g}$ & B & 354.13 & 348 \\
\hline$B_{g}$ & $\mathrm{~S}$ & 369.16 & 370 \\
\hline$A_{g}$ & B & 372.54 & טוכי \\
\hline$B_{g}$ & B & 394.46 & 384 \\
\hline$A_{g}$ & B & 404.53 & 400 \\
\hline$A_{g}$ & B & 419.58 & 424 \\
\hline$B_{g}$ & $\mathrm{~S}$ & 424.26 & 424 \\
\hline$A_{g}$ & $\mathrm{~S}$ & 439.35 & - \\
\hline$A_{g}$ & $\mathrm{~S}$ & 766.73 & - \\
\hline$B_{g}$ & $\mathrm{~S}$ & 779.05 & - \\
\hline$A_{g}$ & B & 865.92 & 853 \\
\hline$B_{g}$ & $\mathrm{~S}$ & 885.50 & 873 \\
\hline$A_{g}$ & $\mathrm{~S}$ & 912.61 & 909 \\
\hline$B_{g}$ & $\mathrm{~S}$ & 923.87 & - \\
\hline$A_{g}$ & $\mathrm{~S}$ & 962.37 & - \\
\hline$A_{g}$ & $\mathrm{~S}$ & 974.69 & - \\
\hline
\end{tabular}


Table S3. Calculated bond distances for $\mathrm{M}-\mathrm{O}\left(\mathrm{M}=\mathrm{Mg}\right.$, Mo) bond paths in $\mathrm{MgMoO}_{4}$ models.

\begin{tabular}{|c|c|c|c|c|c|c|c|}
\hline \multicolumn{8}{|c|}{ Bond Distances M-O-Bulk } \\
\hline \multicolumn{8}{|c|}{ Singlet } \\
\hline \multicolumn{2}{|c|}{$\operatorname{Mg}(1)$} & \multicolumn{2}{|c|}{$\operatorname{Mg}(2)$} & \multicolumn{2}{|c|}{ Mo(1) } & \multicolumn{2}{|c|}{ Mo(2) } \\
\hline 2.034 & $2 x$ & 2.037 & $1 x$ & 1725 & $2 \mathrm{y}$ & 1.732 & $2 \mathrm{x}$ \\
\hline 2.063 & $2 x$ & 2.063 & $2 x$ & 1.155 & $2 x$ & 1.757 & $1 x$ \\
\hline 2.164 & $2 \mathrm{x}$ & $\begin{array}{l}2.118 \\
2.140\end{array}$ & $\begin{array}{l}2 x \\
1 x\end{array}$ & 1.802 & $2 \mathrm{x}$ & 1.840 & $1 x$ \\
\hline \multicolumn{8}{|c|}{ Excited singlet $\left(s^{*}\right)$} \\
\hline \multicolumn{2}{|c|}{$\operatorname{Mg}(1)$} & \multicolumn{2}{|c|}{$\operatorname{Mg}(2)$} & \multicolumn{2}{|c|}{ Мo(1) } & \multicolumn{2}{|c|}{ Mo(2) } \\
\hline 2.034 & $2 \mathrm{x}$ & 2.037 & $1 x$ & & & 1.732 & $2 x$ \\
\hline 2.063 & $2 \mathrm{x}$ & 2.063 & $2 \mathrm{x}$ & 1.700 & $2 x$ & 1.757 & $1 x$ \\
\hline 2.161 & $2 \mathrm{x}$ & $\begin{array}{l}2.118 \\
2.140\end{array}$ & $\begin{array}{l}2 x \\
1 x\end{array}$ & 1.852 & $2 \mathrm{x}$ & 1.839 & $1 x$ \\
\hline \multicolumn{8}{|c|}{ Excited triplet $\left(t^{*}\right)$} \\
\hline \multicolumn{2}{|c|}{$\operatorname{Mg}(1)$} & \multicolumn{2}{|c|}{$\operatorname{Mg}(2)$} & \multicolumn{2}{|c|}{ Mo(1) } & \multicolumn{2}{|c|}{ Mo(2) } \\
\hline 2.017 & $2 \mathrm{x}$ & 2.058 & $1 x$ & 1701 & $2 \mathbf{y}$ & 1.729 & $2 \mathrm{x}$ \\
\hline 2.053 & $2 \mathrm{x}$ & 2.053 & $2 \mathrm{x}$ & 1.191 & $2 \mathrm{X}$ & 1.764 & $1 x$ \\
\hline 2.152 & $2 \mathrm{x}$ & $\begin{array}{l}2.078 \\
2.154\end{array}$ & $\begin{array}{l}2 x \\
1 x\end{array}$ & 1.809 & $2 \mathrm{x}$ & 1.838 & $1 x$ \\
\hline
\end{tabular}

